A. Albu-Rghaif and I. A. Lami, "Novel dictionary decomposition to acquire GPS signals using compressed sensing," presented at the 2014 World Congress on Computer Applications and Information Systems (WCCAIS), Hammamet, Tunisia, 2014.

(C) 2014 IEEE. Personal use of this material is permitted. Permission from IEEE must be obtained for all other uses, in any current or future media, including reprinting/republishing this material for advertising or promotional purposes, creating new collective works, for resale or redistribution to servers or lists, or reuse of any copyrighted component of this work in other works. 


\title{
Novel Dictionary Decomposition to Acquire GPS Signals Using Compressed Sensing
}

\author{
Ali Albu-Rghaif and Ihsan Alshahib Lami \\ Applied Computing Department \\ The University of Buckingham, UK \\ first.last@buckingham.ac.uk
}

\begin{abstract}
When acquiring any wireless signal such as GPS, there is a strong association between the amount of integration, correlation or matching performed by the receiver with the strength of the signal, depending on the acquisition technique used. The Compressive Sensing technique (CS) does reduce the computation by up to $60 \%$ over correlators based method, yet still maintains acquisition integrity. This paper proposes a new method for implementing the dictionary matrix required for $\mathrm{CS}$ and achieving a further saving of more than $80 \%$ in the signal acquisition process without loss of the integration between the code and frequency irrespective of the signal strength. This is achieved by removing the generated Doppler shifts from the "overcomplete" dictionary matrix, while keeping the carrier frequency fixed for all these generated shifted SV codes. Our MATLAB simulation for various signal condition scenarios proves that up to $90 \%$ of computational complexity and memory space can saved.
\end{abstract}

Keywords - dictionary decomposition; GPS signal acquisition; compressive sensing

\section{INTRODUCTION}

In typical signal acquisition processing, the signal is sampled based on Nyquist rate to recover the full information of the signal, if present. CS is a technique to allow recovery of the full signal but with using below-Nyquist rate sampling, if the signal has a sparse representation. The sampling rate is typically equal to the information band of the acquired signal to ensure no loss of information. Therefore CS achieves reduced processing time, due to reduced dimension of the sampled signal (i.e. reduced number of required correlators). This is why CS is very attractive in applications such image and signal processing [1].

For any k sparse received signal $\mathrm{x}$, in some "sparsity basis" $\Psi$ or measurement matrix, then, in CS theory if this signal vector $\mathrm{x} \in \mathrm{R}^{\mathrm{n}}$ can be recovered from $\mathrm{m}$ linear measurements $y \in R^{m}$, where $(k<m<n)$ and is $(m \times n)$ [2], and $y=x$. To achieve this measurement's reduction, the following conditions have to be met [3]: 1) The transform matrix $\Phi$ should satisfy the Restricted Isometry Property (RIP). Note that the RIP is dependent on the orthogonality of the arbitrary subsets in the column vectors of $\Phi$. 2) There should low coherence between the transformation matrix $\Phi$ and the basis matrix. This low coherence, $\mu(\Phi, \Psi)$, is expressed by:

$$
\mu(\Phi, \Psi)=\sqrt{\mathrm{n}} \max _{1 \leq \mathrm{k}, \mathrm{m} \leq \mathrm{n}}\left|\left\langle\varphi_{\mathrm{k}}, \psi_{\mathrm{m}}\right\rangle\right| \in[1, \sqrt{\mathrm{n}}]
$$

The focus of our work is on the acquisition of the GPS 24 SV transmitted signals, but our proposal applies to all type of signals. Our literature survey shows that all CS-based GPS implementations do use the same concept to reconstruct the dictionary matrix $(\Psi)$ : 1) This $\Psi$ matrix consists of the code phased delay and the Doppler frequency shift for each of all these $24 \mathrm{SV}$ (= SV $\times$ Code $\times$ Doppler-Shift $=24 \times 2046 \times 41 \approx$ $2 \times 10^{6}$, where the code phase resolution is half chip and frequency search step is $500 \mathrm{~Hz}$ with range $( \pm 10 \mathrm{KHz})) .2$ ) This $\Psi$ matrix is considered as an overcomplete dictionary (fat dictionary), where the number of columns basis $(\mathrm{C})$ is greater than the length of tested signal $(\mathrm{N})$, or $\mathrm{C}>\mathrm{N}$ [4].

Our implementation achieves the signal acquisition with much less size dimension of the dictionary matrix. This minimisation is achieved by decomposing the parameters of the dictionary matrix to have only shifted codes for all SV, without using any Doppler frequency shifts. Thus we make the acquisition process much faster than the other implementations, resulting in great savings in processing time and memory storage, and therefore much appreciated power consumption especially for Smartphones and Tablets.

Our implementation process, as shown in Fig.1, starts by sampling the received signal according to the chipping rate of $2.046 \mathrm{MHz}$ to satisfy a half chip resolution [5]. Then the sampled signals pass through " $\mathrm{m}$ " Doppler channels simultaneously to generate non-Doppler shift vectors. These vectors are then digitally sensed by the transform matrix $\Phi$. The final stage is to find the dictionary elements, by matching the output from the transformation step with the measurement matrix. Due to the design of our dictionary matrix, we modified the search algorithm. For this, we have chosen to use the "Orthogonal Matching Pursuit" (OMP) algorithm [6], which now search in two dimensions rather than only one dimension. Note that the search in the column vectors in the measurement matrix represents the code phase delay of acquired GPS signals, while the search in row vectors is for the Doppler frequency shift. Moreover, the multi-Doppler channel adopted in our implantation achieves high frequency resolution with much less computational complexity while preserving the signal integrity better than the other methods. Note that the number of row vectors in our transform matrix is equivalent to the number of Doppler channels; in other word, the control parameter is the number of Doppler channels. 


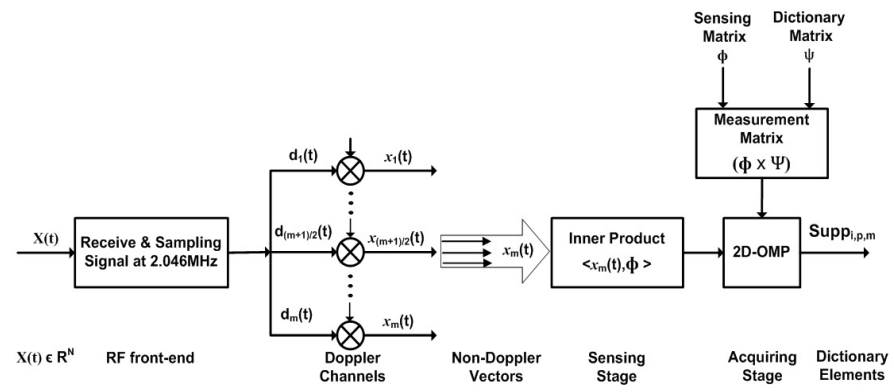

Fig. 1. GPS signal acquisition structure

MATLAB simulation based various signal strength scenarios has shown that our computational complexity, memory requirements and the performance is vastly better than other methods. The results show that our decompose dictionary method obtains enormous saving in terms of implementation, acquisition time.

The rest of the paper has been organized as follows. Section II summarise previous acquisition methods based on CS technique. Section III shows the mathematical model of the GPS signals and decomposed dictionary matrix. Section IV explains the generating non-Doppler vectors and also the two dimension search of dictionary elements. Section V shows the results and the performance of the signal scenarios that have been tested in our proposed model and the comparison between our method and the previous method in terms of computational complexity and memory requirements. Section VI concludes this proposal work with feature work.

\section{RELATED WORK}

Acquiring GPS signals based on CS would save valuable processing time, due to sampling the received signals below Nyquist rate and the capability of acquiring signals consuming few measurements.

The GPS signals can be sampled at a rate equal to "double the chipping rate" $(2.046 \mathrm{MHz})$ so to achieve half chip resolution that can correlated with the dictionary matrix (bank of correlators) to generate sparse vector. This then, using deterministic measurement matrix such as Walsh-Hadamard matrix, to acquire the signal [5]. In the same vein, the measurement matrix can be made to acquire either binary phase shifting key modulation signal (BPSK) or binary offset carrier modulation signal (BOC) [7] . This distinction in signal modulation is realized by adding an indicator (" 0 " for BPSK and " 1 " for BOC) inside the measurement matrix. This method, however, focuses on the chipping rate to acquire the signals without consideration to the codes' length of these signals. For example, both the GPS C/A code signal and the Galileo BOC $(1,1)$ signals have the same chipping rate but they don't have same code length.

Another approach would be to generate sparse vectors of the received GPS signals using Compressive Multichannel Samplers (CMS). In such implementations, the GPS signals are sampled by using random multichannel samplers at the bandwidth information rate $(2.046 \mathrm{MHz})$ to the same half chip resolution [8]. The sparsed signals are then acquired through three steps: 1) convert the sparse vector to a frame using continuous to finite block (CTF) [9] which converts the computational problem from infinite-measurement vectors to multi-measurement vectors, 2) convert these multimeasurement vectors to a single-measurement vector using the "reduce and boost" algorithm (ReMBo) [10], 3) apply the OMP algorithm to find the support values of the acquired signals. However, this method necessitates a complex front-end hardware implementation of such CS receiver. A GPS receiver based on CS (GCSR) has been implemented to overcome the hardware complexity in the RF front-end that has been used in CMS approach as well as to improve the acquisition process [11]. The received GPS signals are sampled at sub Nyquist rate (2.046MHz) via multichannel using deterministic waveform, and then the GPS signals are acquired by using the same process as CMS approach, i.e. using CTF, ReMBo and OMP algorithm to find the support values.

The dictionary matrix (or the bank of correlators) of all mentioned CS implementations is based on code phase delay shifts and Doppler frequency shifts for each of the 24 GPS satellites. This implies that a large dimensional size of this matrix is constructed, and therefore a huge search is required to match the signals for acquisition. In order to reduce the time search of these dictionary elements, the dimension of the dictionary matrix should be reduced. Our proposed implementation decomposes the dictionary matrix into a two stage matrix search, so that only SVs "code shifts" are searched instead of both "codes and Doppler shifts" together. See section IV for full details of our decomposition steps.

\section{DECOMPOSED GPS-DICTIONARY STRUCTURE}

In our work we are primarily concerned with the GPS-L1C/A code signal. The GPS satellite transmits the data navigation message at rate $\left(R_{d}\right) 50 \mathrm{bps}$, and let $d_{i} \in\{\mp 1\}^{N_{d} \times 1}$ be the binary information vector belonging to the $i$ satellites with $N_{d}$ data bits generated at duration $T_{d}=1 / R_{d}=20 \mathrm{~ms}$.

$$
d_{i}(t)=\sum_{n_{d}=0}^{N_{d}-1} d_{i}\left[n_{d}\right] \operatorname{rect}\left(\frac{t-n_{d} T_{d}}{T_{d}}\right), \quad 0 \leq t \leq N_{d} T_{d}
$$

The C/A code uses to spread the satellites' data and also enables the GPS receiver to identify the satellite number, which means each satellite is assigned to a unique $\mathrm{C} / \mathrm{A}$ code. For that reason the $\mathrm{C} / \mathrm{A}$ code considers as a key parameter to construct the dictionary matrix for the GPS-L1 signal. The C/A code waveform is similar to the data waveform $c_{i} \in\{\mp 1\}^{N_{c} \times 1}$ but at a much higher rate $\left(R_{c}\right) 1.023 \mathrm{MHz}$, the code length is 1,023 chips repeated every $1 \mathrm{~ms}$, and the chip duration is $T_{c}=$ $1 / R_{c} \approx 977 n s$.

$$
c_{i}(t)=\sum_{n_{c}=0}^{N_{c}-1} c_{i}\left[n_{c}\right] \operatorname{rect}\left(\frac{t-n_{c} T_{c}}{T_{c}}\right), \quad 0 \leq t \leq N_{c} T_{c}
$$

The spreading code $S_{i}(t)$ of the GPS signal is based on code division multiple access technique, i.e. each GPS satellite has own code, as well each GPS satellite transmits a unique navigation message.

$$
S_{i}(t)=d_{i}(t) \oplus c_{i}(t), 0 \leq t \leq N_{d} T_{d}
$$

where $\oplus$ the modulo-2 adder and $N_{d} T_{d}=N_{c} T_{c}$. 
The spreading code then modulates with L1 carrier frequency that centred at $1575.42 \mathrm{MHz}$ using BPSK modulation. The mathematical representation of the GPS signals is expressed in (4):

$$
y_{i}(t)=\sum \sqrt{2 P}\left[S_{i}(t) \cos \left(\omega_{L 1} t\right)\right]
$$

where $y_{i}(\mathrm{t})$ represents the signals transmitted from $i^{\text {th }}$ satellite $(\mathrm{i}=1 \ldots 24), \mathrm{P}$ is the power of the transmitted signal and $\omega_{\mathrm{L} 1}$ is the carrier frequency of L1.

The GPS L1-C/A signals are received with specific Doppler shift, code phase delay and might the signal comes as a line of sight signal (LOS), or reflected form an obstacle/ obstacles, which called multipath signal. The received GPS signals with/without multipath signal from different satellites can be expresses as:

$$
\begin{gathered}
x(t)=\sum_{i=1}^{I} \sum_{k=0}^{K} \sqrt{2 P_{i, k}} \delta_{i, k}\left(t-n T_{c}-\tau\right) e^{j\left(\omega_{d_{i, k}} t+\theta_{i, k}\right)} \\
+v(t)
\end{gathered}
$$

where $x(t), P, \tau, \omega_{d}, \theta, I, K$ and $v(t)$ represent the received signal, power of received signal, code phase delay, carrier frequency with Doppler shift, carrier phase, LOS signals from $\mathrm{i}=1$ to $\mathrm{I} \in 24$, multipath signals and the complex AWGN respectively.

As shown above in ( $4 \& 5)$, in order to acquire GPS signals it is necessary to know the coarse values of the code phase delay and the Doppler shift frequency in the received signals. Therefore, a huge number of correlators are required to acquire the GPS signals. Our proposal implementation is designed to reduce these correlators, by decomposing the parameters of the dictionary matrix with maintaining signal integrity, i.e. code and frequency. For the sake of clarity on how to decompose the dictionary matrix, let's assume that one of these signals' parameters is fixed or have zero shifts (code phase or Doppler frequency). Then the dimension of the problem (correlators) will be sharply reduced by a factor equal to the fixed parameter. In our work the dictionary matrix decomposes these correlators by making the carrier frequency fixed for all generated shifted codes (2). Consequently, the decomposed dictionary matrix will be represented as a bank of codes I $\mid \mathrm{P}$ multiplied by the same carrier frequency, where $(\mathrm{P})$ is the "search step of code phase delay". As a result our dictionary implementation achieves a massive dimension reduction. Where the number of columns vectors in previous dictionary matrix are $\left(\mathrm{IPQ}=24 \times(1023 \times 2) \times 41 \approx 2 \times 10^{6}\right)$, our implementation on the other hand propose (IP $=24 \times(1023 \times 2)$ $\approx 4.9 \times 10^{3}$ ).

$$
\Psi(\mathrm{t})=\left[\begin{array}{c}
\mathrm{C}_{1,1} e^{j w_{L 1} T_{S}} \\
\mathrm{C}_{1,2} e^{j w_{L 1} T_{S}} \\
\vdots \\
\mathrm{C}_{1,2046} e^{j w_{L 1} T_{S}} \\
\vdots \\
\mathrm{C}_{24,2046} e^{j w_{L 1} T_{s}}
\end{array}\right]^{\mathrm{T}}
$$

$$
\mathrm{C}_{\mathrm{i}, \mathrm{p}}=\sum_{\mathrm{i}=1}^{\mathrm{I}} \sum_{\mathrm{p}=0}^{\mathrm{P}-1} \mathrm{c}_{\mathrm{i}}\left(\mathrm{t}-\mathrm{pT}_{\mathrm{s}}\right)
$$

It is worthwhile to mention that our dictionary matrix is same as a Toeplitz matrix, since all descending diagonal from left to right is constant. Therefore, the solution will be easier to find the right code. In other words, if we assume that the dimension of the dictionary matrix is $(c \mathbf{x} c)$ then the solution will be reduce from $O\left(c^{2}\right)$ to $O(2 c-1)$.

\section{NON-DOPPLER VECTORS GENERATION}

Signal acquisition based CS reduces the number measurements and the number of required correlators, due to sampling the received signal according to either the "occupation information band" or the "information rate", and changing the dimension search from the number of samples to the number of rows in the transform matrix. However, the main obstacle in this acquisition is the large size of dictionary matrix. Usually, the dictionary matrix must include all shifts of codes and frequencies; otherwise the signal can't be acquired.

Before acquiring GPS signal in CS framework, the sampled signal passes through " $m$ " Doppler channels $d_{m}(t)$ simultaneously (8). This process will generate non-Doppler shift signals; also the use of these channels will compensate the measurements in the decomposed dictionary matrix.

$$
d_{m}(t)=e^{j 2 \pi m \Delta f t}
$$

As shown in Fig.2 these channels contain a range of Doppler frequency shifts. When the Doppler shift in the received signal matches the generating frequency of the channel, then the output will contain only the GPS signal without Doppler shift (9), which is easily acquired in CS process.

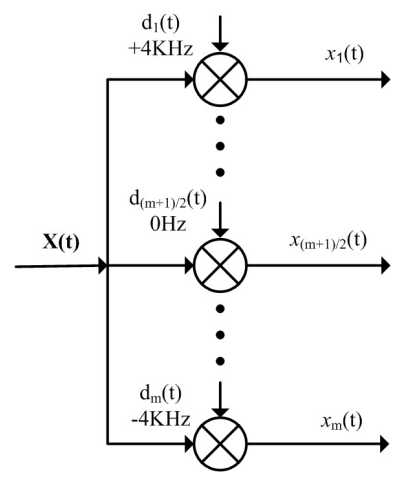

Fig. 2. Multi-Doppler channels

$$
\begin{aligned}
& x_{m}(t)=X(t) d_{m}(t) \\
& x_{m}(t)=\mathcal{S}(t-\tau) e^{j 2 \pi\left(f_{L 1}-f_{d}\right) t} e^{j 2 \pi m \Delta f t} \\
& x_{m}(t)=\mathcal{S}(t-\tau) e^{j 2 \pi f_{L 1} t} e^{j 2 \pi\left(m \Delta f-f_{d}\right) t} \\
& x_{m}(t)=X_{o}(t-\tau) e^{j 2 \pi\left(m \Delta f-f_{d}\right) t} \\
& x_{m}(t)=X_{o}(t-\tau), \quad \text { when } m \Delta f=f_{d}
\end{aligned}
$$

Then the output from the multi-Doppler channels process will be multiplied (inner product) by the transform matrix $\Phi$, 
and the total multiplication will produce a sparse matrix with $(m \times m)$ dimension:

$$
Z \triangleq\left\langle\Phi, x_{m}\right\rangle
$$

The proposal method uses Discrete Fourier Transform (DFT) matrix as a transform matrix with $(m \times n)$ dimension where $m$ is equal to the number of Doppler channels and $n$ is the number of samples.

The acquisition is accomplished by solving matrix V (12), where $\mathcal{D}$ is the measurement matrix. The measurement matrix constructs by multiplying the transform matrix $\Phi(\omega)$ with the decomposed dictionary matrix $\Psi(\omega)$.

$$
\begin{aligned}
& \mathcal{D}=\Phi \Psi \\
& Z=\mathcal{D} \mathrm{V}
\end{aligned}
$$

As shown in (11), in this work not only the size of the dictionary matrix is less, also the size of the measurement matrix is much less than the CMS method in both dimensions. Where the rows vectors, (number of Doppler channels or rows vectors in transform matrix), in which chosen in our work are one sixth (81-channels) of the number of the compressive channels (480-channels), as well as the number of columns vector are also much less as mentioned earlier (IP $<<$ IPQ).

To solve matrix $\mathrm{V}$ a $2 \mathrm{D}$ orthogonal matching pursuit (2DOMP) algorithm has been used to find the support values $\left(\operatorname{supp}_{i, p, m}\right)$. Typically, the OMP algorithm select one item at a time, this item is the support value of $\Psi$ and represents the highest inner product between $\mathcal{D}$ and the residual (where the initial value of the residual is $Z$ ). So, the right code phase delay are determined form the inner product $S_{i, c}=\operatorname{supp}(V)$, while the Doppler shift frequency is calculated by finding the maximum value inside the highest row $S_{d}=\max \left(S_{i, c}\right)$. Since the OMP algorithm takes the transpose of the measurement matrix to be multiply with the residual, then all the columns in the measurement matrix are become rows and vice versa, and the highest matching between them calculates through finding the maximum of the summation rows, as shown below in algorithm 1 .

Algorithm 1: (2D-OMP Algorithm)

1. Initialise $\mathrm{r}^{0}=\mathrm{Z}, \mathrm{y}^{0}=0, \Gamma^{0}=($

2. for $\mathrm{n}=1 ; \mathrm{n}:=\mathrm{n}+1$ till stopping criterion is met
(a) $\nabla^{n}=\Psi^{T} r^{n}$
(b) $p^{n}=\arg _{k} \max \left|\nabla_{\mathrm{p}}\right|$
(c) $q^{n}=\arg _{k} \max \left|\nabla_{\mathrm{p}}^{\mathrm{n}}\right| \quad$ (update step)
(d) $\Gamma^{n}=\Gamma^{n-1} \cup p^{n}$
(e) $y^{n}=\Psi_{\Gamma^{n}}^{\dagger} Z$
(f) $r^{n}=Z-\Psi y^{n}$

3. Output: $p^{n}, q^{n}, r^{n} \& y^{n}$

Here the dagger $\dagger$ indicates the Moore-Penrose
pseudoinverse [12].

\section{EXPERIMENTAL RESULTS AND PERFORMANCE}

In order to highlight the reduction of both computational complexity and memory requirements our proposed method, as shown in Fig.1, has been compared with the MCS [8] and GCSR [11] methods.

Table I illustrates the breakdown of the computational complexity and table II shows the memory requirements for all matrices that are used to accomplish this work. In these tables, "N" represents the 8184 samples for the tested 4ms length signal at $2.046 \mathrm{MHz}$ sampling frequency, "I" is the 24 GPS satellites, "M" is the 81 Doppler channels used in our method, " $C$ " represents the chosen 480 number of channels, " $Q$ " is the 17 frequency search steps as used in CMS and GCSR methods, " $\mathrm{S}$ " is the 24 number of iterations setting, and "P" is the 2046 code phase resolution. This comparison simulation shows that our computational complexity and memory requirements are less by more than $90 \%$. Also, our method satisfies acquiring signals at higher frequency resolution up to $100 \mathrm{~Hz}$, which is nearly equivalent to the "fine frequency" stage of acquisition (reduced search space integration deployed after acquiring the

\begin{tabular}{|c|c|c|}
\hline Steps & $\begin{array}{c}\text { Our Decomposed } \\
\text { Dictionary Method }\end{array}$ & $\begin{array}{l}\text { CMS \& GCSR } \\
\text { Methods }\end{array}$ \\
\hline & $\begin{array}{c}\text { Generate Vectors } \\
\rightarrow \boldsymbol{O}(\mathrm{NM})\end{array}$ & $\begin{array}{c}\text { Digital Compression } \\
\rightarrow \boldsymbol{O}(\mathrm{NC})\end{array}$ \\
\hline & $\begin{array}{l}\text { Inner Product } \\
\qquad \boldsymbol{O}\left(\mathrm{M}^{2}\right)\end{array}$ & $\begin{array}{l}\text { CTF Block } \\
\rightarrow \boldsymbol{O}\left(\mathrm{C}^{2}\right)\end{array}$ \\
\hline Residual Update & \multicolumn{2}{|l|}{$\boldsymbol{O}\left(\mathrm{S}^{2}\right)$} \\
\hline \multirow[t]{3}{*}{ Inner Projection } & $\boldsymbol{O}$ (MIPS) & $\boldsymbol{O}$ (CIPQS) \\
\hline & $\begin{array}{c}\text { Find Code Phase Delay } \\
\rightarrow \boldsymbol{O}(\mathrm{S} \log (\mathrm{IP}))\end{array}$ & \multirow[t]{2}{*}{$\begin{array}{c}\text { Find Dictionary Element } \\
\rightarrow \boldsymbol{O}(\mathrm{S} \log (\mathrm{IPQ}))\end{array}$} \\
\hline & $\begin{array}{l}\text { Find Doppler Shift } \\
\qquad \begin{array}{l}\rightarrow(\mathrm{S} \log (\mathrm{M}))\end{array}\end{array}$ & \\
\hline Stopping Criterion & $\boldsymbol{O}$ (MS) & $\boldsymbol{O}(\mathrm{CS})$ \\
\hline
\end{tabular}
signal) in the traditional receivers.

TABLE I. BREAKDOWN COMPUTATIONAL COMPLEXITY

TABLE II. MEMORY REQUIREMENTS

\begin{tabular}{|l|l|l|l|}
\hline \multicolumn{1}{|c|}{ Matrix type } & \multicolumn{1}{|c|}{$\begin{array}{c}\text { Our Decomposed } \\
\text { Dictionary Method }\end{array}$} & $\begin{array}{c}\text { CMS } \\
\text { Method }\end{array}$ & $\begin{array}{c}\text { GCSR } \\
\text { Method }\end{array}$ \\
\hline Sensing Matrix & MN & C IPQ & C N \\
\hline Dictionary Matrix & N IP & N IPQ & N IPQ \\
\hline Measurement Matrix & M IP & N IPQ & C IPQ \\
\hline \multicolumn{2}{|l|}{ Note that shaded cells represent the lowest memory } \\
\hline
\end{tabular}

Four scenarios of GPS signal condition are simulated using MATLAB as shown in table III. Fig.3 shows the simulation setup the signals are fed into an additive white Gaussian noise channels (AWGN). These signals are then pass to an $2 \mathrm{MHz}$ bandpass filter centred at L1 frequency, an $35 \mathrm{~dB}$ low noise amplifier, and an 10-bit analogue-to-digital converter at sampling rate $2.046 \mathrm{MHz}$ and $3 \mathrm{~dB}$ cascade noise figure. 


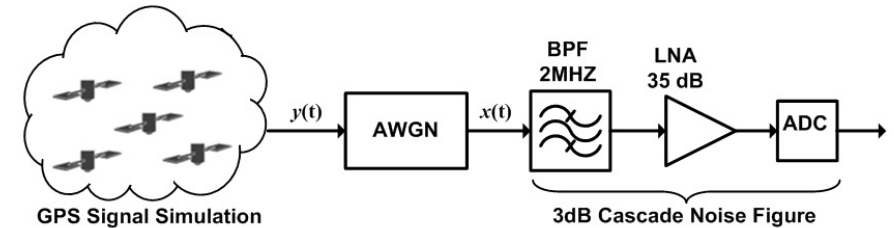

Fig. 3. MATLAB simulation setup

TABLE III. GPS SIGNALS SCENARIOS

\begin{tabular}{|l|l|}
\hline \multirow{2}{*}{$\mathbf{C} \backslash \mathbf{N}$ dB-Hz } & \multicolumn{1}{|c|}{ LOS \& Multipath signals Scenarios } \\
\cline { 2 - 2 } & \multicolumn{1}{|c|}{ Number of received signals } \\
\hline 50 & 5 LOS signals \\
\hline 45 & 5 LOS signals and one multipath for each signal \\
\hline 40 & 5 LOS signals and two multipath for each signal \\
\hline 35 & 5 LOS signals and three multipath for each signal \\
\hline
\end{tabular}

To demonstrate the performance of our proposed method, we have performed several experiments that use different number of Doppler channels to illustrate the achieved performance. Experimentally, we have found that the minimum number of channels that can be used to acquire GPS signal is 33 channels. Increasing this number will enhance the acquisition rate. Therefore, two numbers of Doppler channels have been chosen $(33 \& 81)$ for these simulations. Also, to overcome the length of simulated signal ambiguity, or the number of chosen samples, $1 \mathrm{~ms} \& 4 \mathrm{~ms}$ length signals (or 2046 $\& 8184$ samples respectively) are selected.

As shown in Fig. 4, our implementation achieves low acquisition rate if the minimum number of channels (33channels) is used at minimum signal length (1ms). Increasing the length of tested signal to $4 \mathrm{~ms}$ improves the acquisition rate by $10 \%$. While, when the number of Doppler channels is increased from 33 to 81 channels, the probability of acquiring the $1 \mathrm{~ms}$ signal will increase to $40 \%$. But when also increasing the signal length to $4 \mathrm{~ms}$, the fix rate is more than $20 \%$ overall better performance.

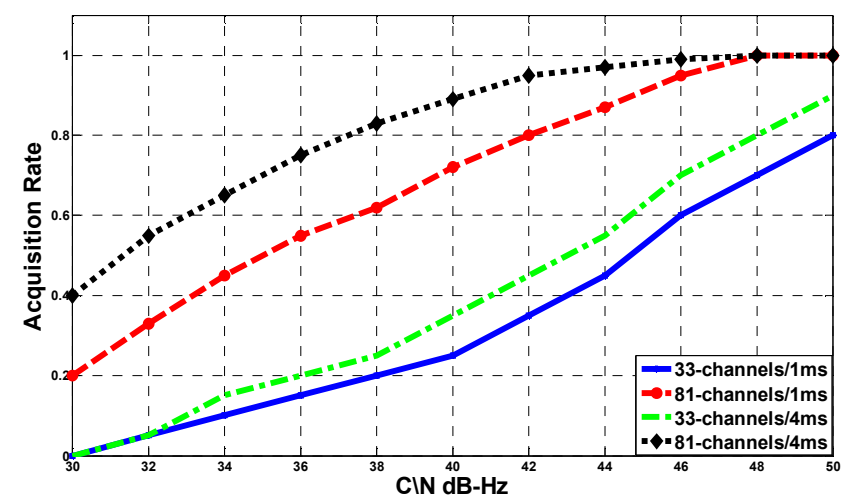

Fig. 4. Acquisition rate for sampling frequency $2.046 \mathrm{MHz}$

\section{CONCLUSION}

Our decomposed dictionary implementation achieves better GPS signals acquisition of at much reduced computational processing by up to $90 \%$ (or $80 \%$ reduction in dictionary matrix size) than other CS-based methods. This reduction makes our implementation method faster and therefore consumes less battery power than other methods.

The control bottleneck of our method is the number of the chosen Doppler channels, i.e. increasing or decreasing these channels will directly effect on the acquisition performance. In another word, increasing the Doppler channels will increase the chance of acquiring available GPS signal; through increasing number of rows in the transform matrix that means increasing the chance of sensing the samples. This will also increase the high frequency resolution that will be close to the "fine frequency" acquisition.

\section{ACKNOWLEDGMENT}

This research is supported by a government scholarship from Iraqi ministry of higher education.

\section{REFERENCES}

[1] Y. C. Eldar and G. Kutyniok, Compressed sensing: theory and applications, Cambridge University Press, 2012.

[2] S. Kirolos, J. Laska, M. Wakin, M. Duarte, D. Baron, T. Ragheb, Y. Massoud and R. Baraniuk, "Analog-to-information conversion via random demodulation," in Design, Applications, Integration and Software, 2006 IEEE Dallas/CAS Workshop on, 2006.

[3] E. J. Candes and M. B. Wakin, "An introduction to compressive sampling," Signal Processing Magazine, IEEE, vol. 25, no. 2, pp. 2130,2008 .

[4] T. L. Hansen, D. Johansen, P. Jorgensen, K. F. Trillingsgaard, T. Arildsen, K. Fyhn and T. Larsen, "Compressed sensing with rank deficient dictionaries," in Global Communications Conference (GLOBECOM), 2012 IEEE, 2012.

[5] S. Kong, "A Compressed Sensing Technique for GNSS Signal Acquisition," in Proceedings of the 2012 International Technical Meeting of The Institute of Navigation, 2012.

[6] Y. C. Pati, R. Rezaiifar and P. Krishnaprasad, "Orthogonal matching pursuit: Recursive function approximation with applications to wavelet decomposition," in Signals, Systems and Computers, 1993. 1993 Conference Record of The Twenty-Seventh Asilomar Conference on, 1993.

[7] S.-H. Kong, "A Deterministic Compressed GNSS Acquisition Technique," 2013.

[8] X. Li, Y. C. Eldar and A. Scaglione, "Low complexity acquisition of GPS signals," in Signal Processing Advances in Wireless Communications (SPAWC), 2011 IEEE 12th International Workshop on, 2011.

[9] M. Mishali and Y. C. Eldar, "Blind multiband signal reconstruction: Compressed sensing for analog signals," Signal Processing, IEEE Transactions on, vol. 57, no. 3, pp. 993-1009, 2009.

[10] M. Mishali and Y. C. Eldar, "Reduce and boost: Recovering arbitrary sets of jointly sparse vectors," Signal Processing, IEEE Transactions on, vol. 56, no. 10, pp. 4692-4702, 2008.

[11] I. Lami, A. Albu-Rghaif and M. Al-Aboodi, "GCSR: A GPS Acquisition Technique using Compressive Sensing Enhanced Implementation," IJEIT, vol. 3, no. 5, pp. 250-255, 2013

[12] T. Blumensath and M. E. Davies, "Gradient pursuits," Signal Processing, IEEE Transactions on, vol. 56, no. 6, pp. 2370-2382, 2008. 